\title{
Hyperspectroscopic Photoluminescence Imaging of Si Micropillar Arrays
}

\author{
Yang Qian, David Magginetti and Heayoung Yoon \\ University of Utah, Salt Lake City, Utah, United States
}

High-aspect ratio Si pillar arrays are attractive options for chemical and biological sensors owing to their high surface area in comparison to their planar counterparts. In addition to easy production of threedimensional (3D) structures via established microfabrication techniques, Si micropillars can further be tailored to desirable nanostructures for enhanced optical sensing at their interfaces in a media of interest (e.g., liquid, gas). In this work, we describe the fabrication of Si micropillar arrays via metal-assisted chemical etching (MACE) processes and show an optical enhancement at their Si shell in a fluorescence oil environment. The hyperspectral photoluminescence (PL) images collected with a $405 \mathrm{~nm}$ laser beam illumination suggest an unexpected PL emission at $500 \mathrm{~nm}$ can be attributed to a strong light-matter interaction at the interface of nanostructured Si pillars and fluorescence oil.

Si micropillar arrays were fabricated using a conventional lithography technique. First, double-layer photoresists were spin-coated (LOR10B and S1813) on a Si substrate (resistivity $<0.005$ ohm $\times \mathrm{cm}$ ). Arrays of circles were patterned and developed. Following an $\mathrm{O}_{2}$ descum, a thin layer of Au film was evaporated $(\approx 3 \AA / \mathrm{s} ; \approx 30 \mathrm{~nm}$ thick) and lifted off. The Au patterned samples were then dipped into a mixed chemical solution ( $10 \mathrm{M} \mathrm{HF}$ and $0.3 \mathrm{M} \mathrm{H}_{2} \mathrm{O}_{2}$ in DI water) for 2 hours at room temperature. Each micropillar array $(2.5 \mathrm{~mm} \times 2.5 \mathrm{~mm})$ contained $\approx 10^{5}$ pillars. The diameter and height of the pillars were $\approx 8 \mu \mathrm{m}$ and $\approx 20 \mu \mathrm{m}$, respectively. Figure 1a shows a representative Si pillar array. An array of Si pillar sample was filled with oil (low fluorescence oil) and covered with a transparent glass coverslip (Fisher Scientific; $\approx 150 \mu \mathrm{m}$ thick and $35 \mathrm{~mm}$ in diameter). The sample was placed on a stage for PL measurements. As shown in a schematic in Figure 1b, a $405 \mathrm{~nm}$ laser beam source $(\approx 1.5 \mathrm{~mW})$ was irradiated on the surface of $\mathrm{Si}$ pillars through an oil-based objective lens (100X; 1.4 N.A.). The characteristic PL signals were collected by either an avalanche Si photodiode (APD) or a spectrometer (SpectraPro 2300i; Princeton Instruments). For PL images, we scanned an area of $35 \mu \mathrm{m} \times 35 \mu \mathrm{m}(64$ pixels $\times 64$ pixels $)$ of the pillar array at $0.03 \mathrm{~Hz}(\approx 208 \mu$ s per pixel). Each pixel contains a PL spectrum in a wavelength range from $300 \mathrm{~nm}$ to $890 \mathrm{~nm}$ with a step size of $\approx 0.43 \mathrm{~nm}$. The PL datasets were analyzed by MATLAB and Origin programs.

Figure 2a displays a panchromatic PL image of our Si micropillars in a fluorescence oil. The intense brightness contrast was observed on Si pillars. Figure $\mathbf{2 b}$ plots a set of PL spectra along the line on a single Si pillar (Figure 2a). Two prominent PL peaks were observed at $\approx 665 \mathrm{~nm}(\approx 1.9 \mathrm{eV})$ and $\approx 520 \mathrm{~nm}$ $(\approx 2.4 \mathrm{eV})$. We integrated the PL spectrum at each pixel near the peak of $665 \mathrm{~nm}(550 \mathrm{~nm} \sim 885 \mathrm{~nm})$ and $520 \mathrm{~nm}(318 \mathrm{~nm} \sim 550 \mathrm{~nm})$ and constructed the spectroscopic images in Figure 2c and Figure 2d, respectively. The PL map clearly showed that the red-luminescence $(\approx 665 \mathrm{~nm} ; \approx 1.9 \mathrm{eV})$ is mainly attributed to the surface of the Si pillar. This luminescence is likely attributed to a thin $\mathrm{SiO}_{2}$ layer $(<100$ $\mathrm{nm})$ that was formed during the MACE redox processes [1, 2]. A main contribution of the luminescence appears to be associated with the defects in $\mathrm{SiO}_{2}$, specifically non-bridging oxygen $(\equiv \mathrm{Si}-\mathrm{O} \bullet$ ). In contrast, the peak at $\approx 520 \mathrm{~nm}(2.38 \mathrm{eV})$ can be a convolution of complex photocarrier dynamics of our sample and the PL system (e.g., low fluorescence oil, defect luminescence of coverslip and optical components). We performed a control PL imaging without oil and coverslip. The PL datasets collected with an air-based PL setup (40X objective lens; numerical aperture [N.A.] =0.6) also showed a weak luminesce in this 
wavelength regime, yet the intensity was almost negligible. Our high-resolution scanning electron microscopy (SEM) image showed the presence of nanostructures $(<200 \mathrm{~nm})$ on the micropillars. Considering the prominent luminescence on the shell of Si pillars in the oil-based PL, we speculate a strong light-matter interaction occurred at the interface of Si pillars and fluorescence oil. It has been suggested that the luminescence and near $550 \mathrm{~nm}(2.2 \mathrm{eV})$ are most likely unsaturated bonds in Si (e.g., $\mathrm{Si}_{3} \equiv \mathrm{Si}-$ ) and are located at the $\mathrm{Si}_{-} \mathrm{SiO}_{2}$ interface [3]. Currently, we are investigating PL properties of various luminescence particles in liquid that are in contact with the nanostructures of Si pillars [4].
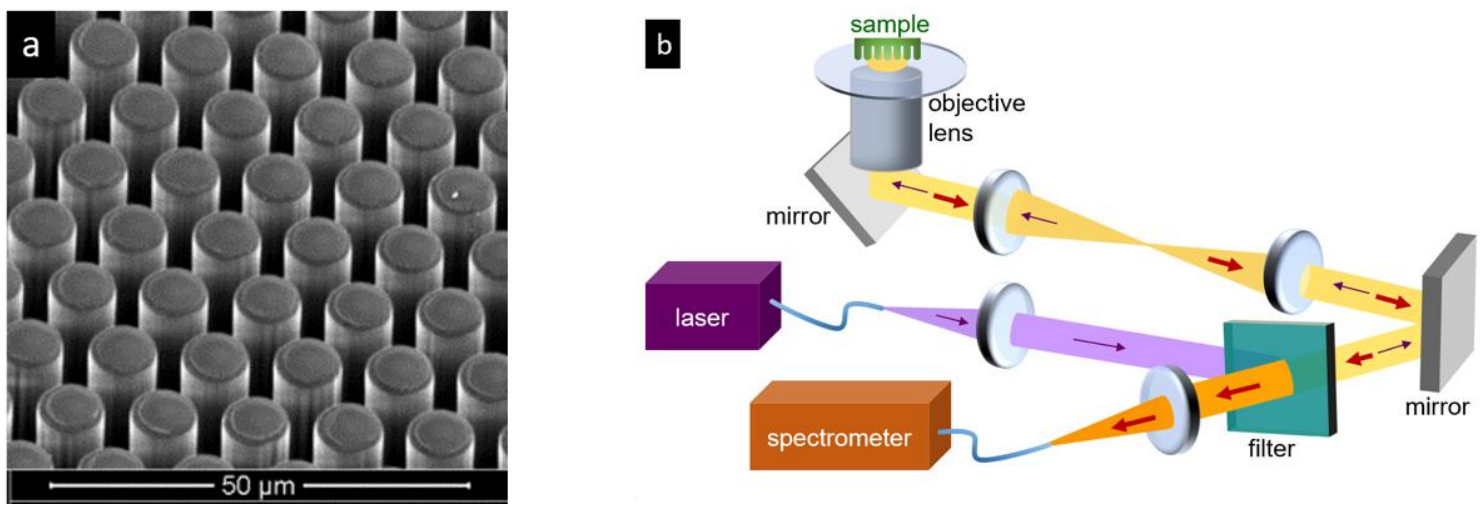

Figure 1. (a) SEM image of a portion of Si micropillar array fabricated by metal-assisted chemical etching (MACE). (b) A schematic of confocal photoluminescence (PL) microscopy set-up. 

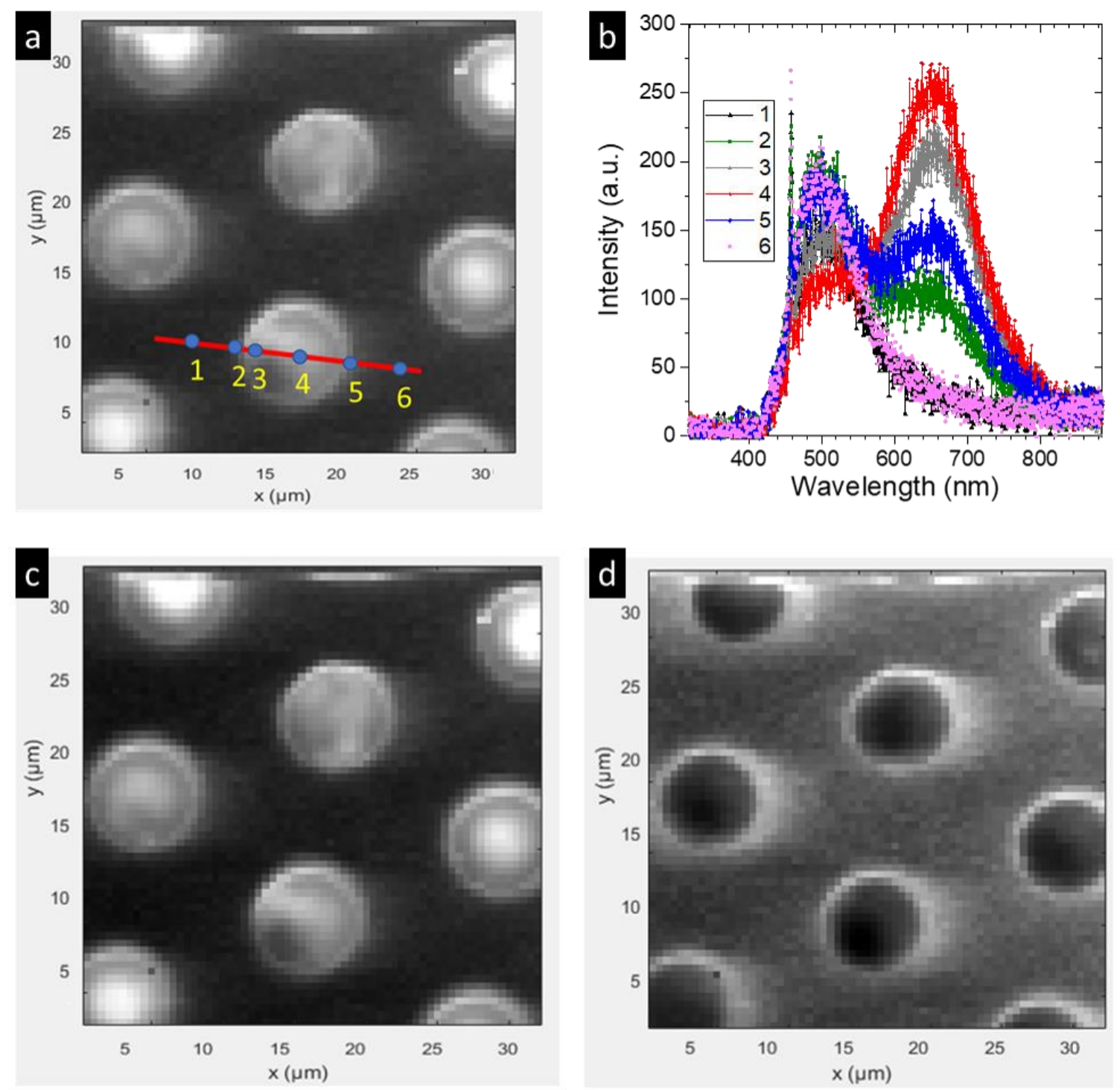

Figure 2. (a) Panchromatic PL imaging of a portion of Si micropillar array (wavelength $=405 \mathrm{~nm}$; power $=1.5 \mathrm{~mW}$ ). (b) PL spectra of the blue points on the red line in Figure 2a. Spectroscopic PL image at (c) $665 \mathrm{~nm}(550 \mathrm{~nm} \sim 885 \mathrm{~nm})$ and (d) $520 \mathrm{~nm}(318 \mathrm{~nm} \sim 550 \mathrm{~nm})$. The brightness contrast corresponds to the integrated intensity of the spectra.

\section{References}

[1] Huang, Z., Geyer, et al., Metal-Assisted Chemical Etching of Silicon: A Review. Adv. Mater. 23, 285308 (2011).

[2] Azeredo, B. P. et al., Direct Imprinting of Porous Silicon via Metal-Assisted Chemical Etching. Adv. Funct. Mater. 26, 2929-2939 (2016).

[3] A.P. Barabana, et al., Luminescence of $\mathrm{SiO} 2$ layers on silicon at various types of excitation, J. of Luminescence 205, 102-108 (2019).

[4] Acknowledgment: This work made use of the University of Utah USTAR shared facilities support, in part, by the MRSEC Program of NSF under Award No. DMR-1121252. This work was performed, in 
part, at the Center for Integrated Nanotechnologies, an Office of Science User Facility operated for the U.S. Department of Energy (DOE) Office of Science by Los Alamos National Laboratory (Contract 89233218CNA000001) and Sandia National Laboratories (Contract DE-NA-0003525). We thank M. Wang and Prof. J. Gerton for their support on the PL measurements, and Dr. J. Lee and D. Stowe for valuable discussions. 